\title{
CIÊNCIANATURA
}

\section{Avaliação dos produtos de evapotranspiração baseados em sensoriamento remoto MOD16 e GLEAM em sítios de fluxos turbulentos do Programa LBA}

\author{
Evaluation of remotely sensed evapotranspiration products MOD16 and GLEAM in eddy \\ covariance flux sites from LBA Project
}

Adriana Aparecida Moreira, Daniela Santini Adamatti e Anderson Luis Ruhoff

Instituto de Pesquisa Hidráulicas - Universidade Federal do Rio Grande do Sul

\section{Resumo}

O objetivo do trabalho foi analisar o desempenho dos conjuntos de dados de evapotranspiração (ET) dos modelos MOD16 e GLEAM em nove sítios nos quais estão instaladas torres de monitoramento de fluxos turbulentos. Para esse fim, os dados de ambos os produtos foram adquiridos e as médias diárias calculadas. As ET estimadas pelos modelos foram comparados com as observadas nas torres de monitoramento de fluxos turbulentos do Projeto de Grande Escala da Biosfera-Atmosfera na Amazônia (LBA). Foi realizada a análise estatística através do coeficiente de correlação (R), do erro médio quadrático (RMSE) e do erro médio (BIAS). Os resultados indicam que os dois produtos de ET analisados são capazes de representar, de forma geral, o comportamento da ET observado nas torres de fluxo. O MOD16 e o GLEAM apresentam estatísticas similares, quando comparados com os dados observados. Tanto as estimativas dos modelos quanto as das torres de fluxo turbulento estão sujeitas à incertezas que influenciam a análise dos produtos de ET.

Palavras-chave: LBA. MODIS. GLEAM. Validação

Abstract

This study aims to evaluate the performance of MOD16 and GLEAM evapotranspiration (ET) datasets in nine eddy covariance monitoring sites. Data from both ET products were downloaded and its daily means calculated. Evapotranspiration estimations were then compared to the observed ET in the eddy covariance monitoring sites from the Large-Scale Biosphere-Atmosphere Experiment in the Amazon (LBA). We performed a statistical analysis using the correlation coefficient (R), the root mean square error (RMSE) and BIAS. Results indicate that, in general, both products can represent the observed ET in the eddy covariance flux towers. MOD16 and GLEAM showed similar values to the calculated statistics when ET estimates were compared to observed ET. Model estimates and eddy covariance flux towers are subject to uncertainties that influence the analysis of remotely-sensed ET products.

Keywords: LBA. MODIS. GLEAM. Validation 


\section{Introdução}

A evapotranspiração (ET) é uma componente importante nos processos e modelos de mudança climática globais, no balanço hídrico, na produtividade primária líquida, e na gestão de eventos extremos. A sua quantificação e previsão se torna difícil devido à influência de diversos fatores, tais como, a heterogeneidade da superfície terrestre, a complexidade dos ecossistemas, e as grandes variações nos processos físicos da interação solo-planta-atmosfera (CHEN et al., 2014; LIAQAT; CHOI, 2017). Além disso, as técnicas existentes visando a estimativa da ET in situ, como o lisímetro e as torres de fluxos turbulentos (Eddy Covariance), apresentam limitações na representação da ET para grandes escalas espaciais (HU; JIA; MENENTI, 2015).

Os modelos de ET com base em sensoriamento remoto têm sido aceitos como alternativas eficazes nas estimativas de evapotranspiração em grande escala. No entanto, estudos são necessários a fim de verificar o quão bem esses modelos são capazes de representar a evapotranspiração em diversas escalas espaciais e temporais (KIM et al., 2012; HU; JIA; MENENTI, 2015). Dentre os produtos de evapotranspiração existentes pode-se citar o MOD16 e o GLEAM.

O MOD16 estima a evapotranspiração com base na equação de Penman-Monteith usando produtos da superfície terrestre do MODIS e um conjunto de dados meteorológicos de reanálise globais, fornecendo uma considerável série temporal de dados de ET para todo o globo (LIAQAT; CHOI, 2017). Já o GLEAM é um conjunto de algoritmos que visa a estimativa de evapotranspiração terrestre (baseado na equação de Priestley e Taylor) e umidade do solo da zona da raiz a partir de dados de sensoriamento remoto (MARTENS et al., 2017).

Neste contexto, e considerando a importância de estimativas precisas de ET para a compreensão da dinâmica do ciclo hidrológico, principalmente em grandes escalas, temporal e espacial, bem como para subsidiar a gestão dos recursos hídricos, este trabalho visou analisar o desempenho dos conjuntos de dados de ET do MOD16 e do GLEAM, em nove sítios, com diferentes coberturas do solo, nos quais estão instaladas torres de monitoramento de fluxos turbulentos, para o período compreendido entre 2000 e 2006.

\section{Material e Métodos}

\section{Descrição dos sítios das Torres de Fluxo}

Neste estudo foram utilizadas torres de fluxo do LBA na Amazônia e uma torre de fluxo no Cerrado, em São Paulo (PDG) (Tabela 1) (Figura 1). O LBA (Large-Scale Biosphere and Atmosphere Experiment in the Amazon) é um programa de pesquisa internacional com objetivo investigar os processos; climatológico, ecológico, biogeoquímico e hidrológico na Amazônia, bem como o impacto das mudanças de uso da terra nestes processos e as interações entre a Amazônia e o sistema terrestre (AVISSAR; NOBRE, 2002; ROBERTS; KELLER; SOARES, 2003).

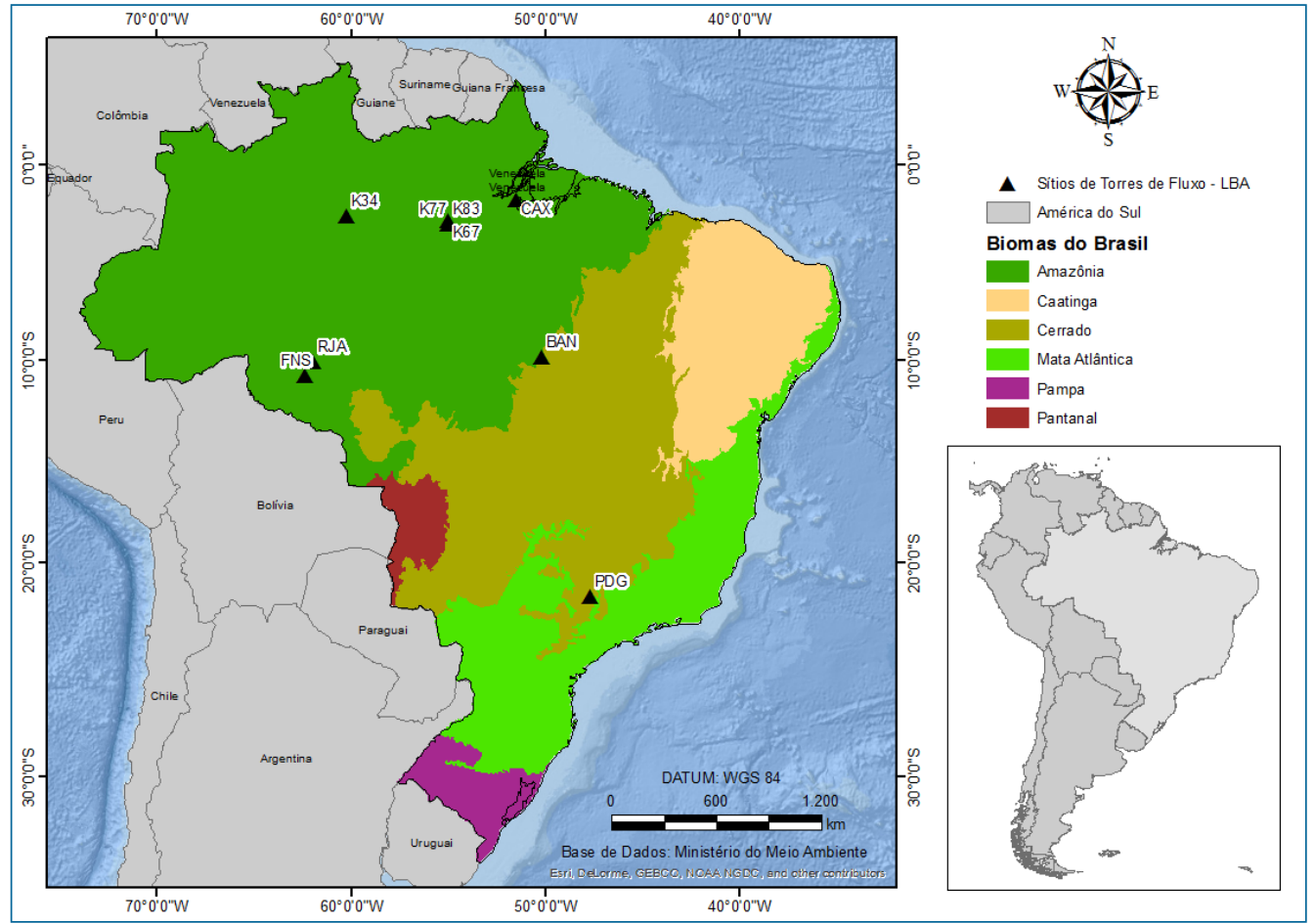

Figura 1 - Localização das torres de fluxo 
Tabela 1 - Descrição das Torres de Fluxos utilizadas

\begin{tabular}{c|c|c|c|c|c}
\hline Sítio & Latitude $\left(^{\circ}\right)$ & Longitude $\left(^{\circ}\right)$ & Localização & Cobertura do Solo & Período das Observações \\
\hline BAN & $-9,82$ & $-50,15$ & Tocantins & Cerrado com inundação sazonal & out 2003 - dez 2006 \\
\hline CAX & $-1,74$ & $-51,45$ & Pará & Floresta Tropical & jan 1999- jul 2003 \\
\hline FNS & $-10,76$ & $-62,35$ & Rondônia & Pastagem & fev 1999 - nov 2002 \\
\hline K34 & $-2,60$ & $-60,20$ & Amazonas & Floresta Tropical & jun 1999- set 2006 \\
\hline K67 & $-2,85$ & $-54,95$ & Pará & Floresta Tropical & jan 2002 - jan 2006 \\
\hline K77 & $-3,02$ & $-54,88$ & Pará & Agricultura/Pastagem & jan 2000 - dez 2005 \\
\hline K83 & $-3,01$ & $-54,97$ & Pará & Floresta Tropical & jun 2000 - mar 2004 \\
\hline PDG & $-21,61$ & $-47,64$ & São Paulo & Cerrado & jan 2004 - dez 2006 \\
\hline RJA & $-10,07$ & $-61,93$ & Rondônia & F. Tropical semidecidual & mar 1999 - nov 2002 \\
\hline
\end{tabular}

\section{MOD16}

O MOD16 (MU et al., 2007; MU; ZHAO; RUNNING, 2011) foi desenvolvido para calcular a ET através da utilização integrada de dados meteorológicos de reanálise e de dados de sensoriamento remoto do sensor MODIS (Moderate Resolution Imaging Spectroradiometer). No algoritmo, a evapotranspiração total ( $\lambda \mathrm{E})$ é particionada em evaporação da água interceptada pelo dossel $\left(\lambda \mathrm{E}_{w c}\right)$, transpiração do dossel $\left(\lambda \mathrm{E}_{t}\right)$ e evaporação do solo $\left(\lambda \mathrm{E}_{s}\right)$, sendo definida como $\lambda \mathrm{E}=\lambda \mathrm{E}_{w c}+\lambda \mathrm{E}_{t}+\lambda \mathrm{E}_{s}$. A contribuição de cada um dos componentes é derivada da equação de Penman-Monteith, ponderada com base na fração de cobertura vegetal, na fração de superfície úmida e na energia incidente disponível. O algoritmo de ET MOD16 gera o produto de evapotranspiração em uma grade regular de 500 x $500 \mathrm{~m}^{2}$, em escala global, em intervalos de 8 dias, mensal e anual.

\section{GLEAM}

O GLEAM (Global Land Surface Evaporation: The Amsterdam Methodology), é um modelo elaborado com a finalidade de usar o máximo de observações derivadas de sensoriamento remoto para estimar o fluxo evaporativo sobre a superfície terrestre, diariamente, em uma escala global e em uma resolução espacial de $0,25^{\circ}$. O modelo separa os diferentes componentes terrestres da evapotranspiração. Cada célula da grade é composta quatro diferentes tipos de cobertura de terrestre, sendo; solo nu; vegetação baixa; vegetação alta e superfície d'água. O fluxo evaporativo é calculado separadamente para cada uma das frações e posteriormente agregado a escala do pixel com base na cobertura fracionada do tipo de cobertura terrestre. A equação de Priestley e Taylor é usada para calcular a evapotranspiração potencial que posteriormente é convertida em estimativas de transpiração real ou evaporação do solo, conforme a cobertura terrestre (MIRALLES et al., 2011; MARTENS et al., 2017).

\section{Procediementos metodológicos}

Foram adquiridos dados com controle de qualidade em intervalo de tempo diário nas nove torres de fluxo, disponibilizados através do Distributed Active Archive Center (https://daac.ornl.gov). Foi realizada uma composição dos dados diários de calor latente (LE) em média de 8 dias, com o intuito de compatibilizar a escala temporal com os dados de sensoriamento remoto. No cálculo das médias de 8 dias a partir dos dados diários, foi utilizado o critério de existência máxima de $25 \%$ de falhas. Para a conversão dos dados de LE (W.m ${ }^{-2}$ ) em dados de ET ( $m m^{-1}$ ) utilizou-se metodologia empregada em Mu, Zhao e Running (2011) utilizando a Equação 1.

$$
E T=\frac{L E}{\lambda}
$$

onde $\lambda$ é o calor latente de vaporização da água (MJ.kg-1). Foram utilizados dados MOD16, coleção 5, de resolução tem- 
poral de 8 dias, para o período de janeiro de 2000 a dezembro de 2006. Dados GLEAM versão 3.0, em escala diária, também foram adquiridos, para o período de janeiro de 2003 a dezembro de 2006. Foram extraídos valores médios de ET para o GLEAM e para o MOD16. A janela para os dados ET MOD16 foi de 3x3 km e a janela de aquisição de valores médios para ET GLEAM foi em um raio de $25 \mathrm{~km}$, abrangendo cerca de 4 pixels da imagem. Os dados de ET GLEAM e MOD16 foram comparados com os dados de ET calculados das torres de fluxo. Estatísticas foram calculadas para avaliar a qualidade das estimativas de ET, sendo calculado o coeficiente de correlação (R), o erro médio quadrático (RMSE) e o erro médio (BIAS).

\section{Resultados e Discussão}

Os resultados apresentados na Tabela 2 mostram que as estimativas de ET do MOD16 apresentaram RMSE variando de $0,32 \mathrm{~mm} \cdot \mathrm{d}^{-1}$ a $0,70 \mathrm{~mm} \cdot \mathrm{d}^{-1}$ para os sítios em área de floresta tropical (CAX, K34, K67 e K83) e coeficiente de correlação variando entre 0,21 e 0,52. A ET estimada pelo GLEAM, para os sítios localizados no mesmo bioma, apresentaram valores de $\mathrm{R}$ variando entre $-0,08$ e 0,52 e os do RMSE entre 0,32 $\mathrm{mmd}^{-1}$ a $0,41 \mathrm{mmd}^{-1}$. Mu, Zhao e Running (2011) obtiveram valores de R de 0,33 e 0,35 e RMSE de $1,28 \mathrm{~mm} . \mathrm{d}^{-1}$ e 1,39 mm.d $\mathrm{d}^{-1}$, para os sítios K67 e K83, respectivamente, utilizando dados do MOD16.

Nos sítios em áreas de pastagem (FNS) e pastagem/agricultura (K77), o MOD16 apresentou RMSE de 1,29 mm.d ${ }^{-1}$ e R de -0,34, em FNS, e RMSE $=0,44 \mathrm{~mm} \cdot \mathrm{d}^{-1}$ e $\mathrm{R}=0,07$, para o sítio K77. Pode-se observar uma melhor correlação, em comparação à obtida para o MOD16, dos dados estimados pelo GLEAM para o sítio K77, no qual foram obtidos R = 0,52 e RMSE $=0,49 \mathrm{~mm} \cdot \mathrm{d}^{-1}$. Conforme Oliveira et al. (2017) o baixo desempenho do MOD16, em áreas de pastagens e áreas agrícolas, pode estar relacionado à sua maior capacidade de representar a evapotranspiração em áreas de floresta do que em áreas de não floresta.

O sítio PDG, localizado em área de Cerrado apresentou estatísticas similares para ambas estimativas de ET, para o $\operatorname{MOD} 16\left(\mathrm{R}=0,84 \mathrm{RMSE}=0,45 \mathrm{~mm} \cdot \mathrm{d}^{-1}\right)$ e para o GLEAM $\left(\mathrm{R}=0,91 \mathrm{RMSE}=0,29 \mathrm{~mm} \cdot \mathrm{d}^{-1}\right)$. Em estudo realizado por Ruhoff et al. (2013), foram obtidos valores de R de 0,78 e um RMSE de 0,78 mm.d ${ }^{-1}$ para o MOD16, para o sítio em questão.

Nos resultados para o sítio BAN as estimativas do MOD16 e do GLEAM apresentaram coeficientes de correlação semelhantes, 0,58 e 0,50, e um RMSE de 1,24 mm.d $\mathrm{d}^{-1}$ e $0,81 \mathrm{~mm} . \mathrm{d}^{-1}$, respectivamente.

No sítio RJA, localizado em área de floresta tropical semidecidual, classificada como Floresta Tropical pelo MOD12Q1, foram obtidos os valores de $\mathrm{R}=0,20$ e $\mathrm{RMSE}=0,66 \mathrm{~mm} \cdot \mathrm{d}^{-1}$, para as estimativas do MOD16.

De uma forma geral, os dados do MOD16 e GLEAM apresentaram subestimativas e superestimavas nos mesmos sítios, com exceção do sítio PDG, onde os valores foram ligeiramente superestimados no MOD16 $\left(0,24\right.$ mm.d $\left.\mathrm{d}^{-1}\right)$ e subestimados no $\operatorname{GLEAM}\left(-0,61 \mathrm{~mm} \cdot \mathrm{d}^{-1}\right)$.

As discrepâncias entre os dados do MOD16 quando comparados com dados observados podem estar atreladas à alguns fatores, como os erros inerentes da classificação imprecisa dos tipos de cobertura da terra e às incertezas associadas aos dados de entrada, tais como a fração fotossinteticamente ativa, o índice de área foliar, e aos dados meteorológicos de reanálise do GMAO (Global Modeling and Assimilation Office) (MU; ZHAO; RUNNING, 2011; KIM et al., 2012).

Além disso, sabe-se que a imprecisão nas medições das torres de fluxo turbulento também pode contribuir para que haja diferenças consideráveis entre os dados de ET observados e os estimados por modelos baseados em sensoriamento remoto (KIM et al., 2012). Imprecisões nas medidas são frequentemente observadas através do não fechamento do balanço de energia, influenciando assim, de forma relevante em como dados devem ser comparados com estimativas realizadas por modelos. De acordo com Wilson et al. (2002), muitos estudos indicam que o fluxo de energia da superfície, que corresponde à soma do calor latente (LE) e do calor sensível (H), são geralmente (mas nem sempre) subestimados em cerca de 10 a 30\% com relação à estimativa da energia disponível (que corresponde à radiação líquida (Rn) menos o fluxo do solo $(\mathrm{G})$ e a taxa de armazenamento (S). Neste sentido Ruhoff (2011) relata que nos sítios do LBA o balanço de energia apresenta um fechamento de 70 a $80 \%$.

Ao analisar a variação de ET média para os dias do ano, verifica-se uma boa representação dos dados de ET estimados pelos modelos MOD16 e GLEAM, em comparação aqueles medidos pelas torres de fluxo (Figura 2).

No sítio BAN durante a estação seca (jun-set), os valores de ET sensoriamento remoto apresentam-se inferiores aos valores da torre de fluxo. No sítio FNS, que é uma área de pastagens, os valores de ET MOD16 e GLEAM, também apresentam subestimados na mesma época. Nos sítios K34, K67, K77 e RJA observa-se comportamento similar das estimativas de ET do MOD16 e do GLEAM, que no geral, apresentaram valores superiores aos observados na torre de fluxo. Nos sítios K83 e PDG são observadas as melhores aproximações das curvas de ET obtidas utilizando os produtos de ET por sensoriamento remoto com aquela gerada com os dados de ET da torre de fluxo. 


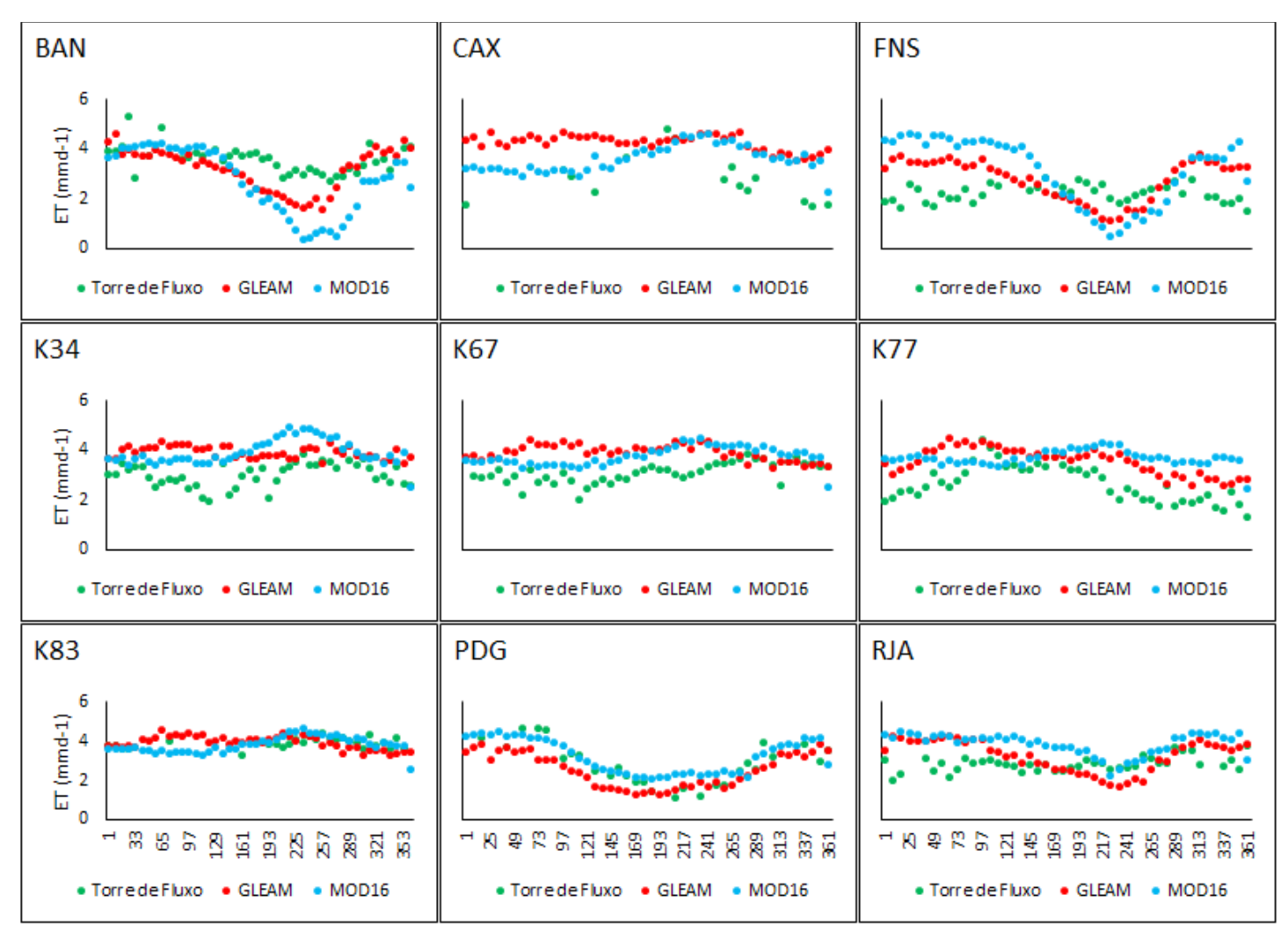

Figura 2 - Evapotranspiração (ET) médio para cada dia do ano, para o período de 2000 a 2006, para MOD16, Torres de Fluxo e GLEAM

Tabela 2 - Estimativas de ET MOD16 e GLEAM em comparação com as medições de ET das torres de fluxo do LBA, entre 2000 a 2006.

\begin{tabular}{c|c|c|c|c|c|c|c|c}
\hline Sítio & \multicolumn{2}{|c|}{ Tamanho amostral } & \multicolumn{2}{|c|}{$\mathrm{R}$} & \multicolumn{2}{c}{ RMSE $\left(\mathrm{mm}^{-1} \mathrm{~d}^{-1}\right.$} & \multicolumn{2}{c}{ BIAS $\left(\mathrm{mm}^{\mathrm{d}}{ }^{-1}\right)$} \\
\hline & MOD16 & GLEAM & MOD16 & GLEAM & MOD16 & GLEAM & MOD16 & GLEAM \\
\hline BAN & 84 & 84 & 0,58 & 0,50 & 1,24 & 0,81 & $-1,28$ & $-0,51$ \\
\hline CAX & 14 & - & 0,52 & - & 0,70 & - & 0,83 & \\
\hline FNS & 58 & - & $-0,34$ & - & 1,29 & - & 0,98 & - \\
\hline K34 & 133 & 83 & 0,30 & 0,32 & 0,68 & 0,32 & 0,88 & 0,99 \\
\hline K67 & 112 & 83 & 0,21 & $-0,08$ & 0,45 & 0,41 & 0,72 & 0,77 \\
\hline K77 & 199 & 119 & 0,07 & 0,52 & 0,44 & 0,49 & 0,95 & 1,01 \\
\hline K83 & 47 & 14 & 0,33 & 0,39 & 0,32 & 0,32 & $-0,03$ & $-0,32$ \\
\hline PDG & 33 & 18 & 0,84 & 0,91 & 0,45 & 0,29 & 0,24 & $-0,61$ \\
\hline RJA & 74 & - & 0,20 & - & 0,66 & - & 0,96 & - \\
\hline
\end{tabular}

\section{Conclusões}

Ao analisar os dois produtos de estimativa de ET gerados com base em dados de satélite, nos nove sítios estudados, observa-se que o MOD16 e o GLEAM apresentaram resultados similares no que se refere às estatísticas obtidas. As médias diárias, calculadas considerando todos os anos analisados, permitiram observar que o MOD16 e o GLEAM são capazes de representar, de forma geral, o comportamento observado nas torres de fluxo turbulento. Ressalta-se que existem diversas incertezas associadas tanto à geração das estimativas através de modelos baseados em sensoriamento remoto, quanto à de dados nas torres de fluxos turbulentos, que influenciam a avaliação dos produtos de ET. 


\section{Agradecimentos}

Os autores agradecem ao programa de pós-graduação em Recursos Hídricos e Saneamento Ambiental, do Instituto de Pesquisas Hidráulicas (IPH) - Universidade do Rio Grande do Sul (UFRGS), ao Conselho Nacional de Desenvolvimento Científico e Tecnológico pelo apoio e incentivo e ao LBA (Large-Scale Biosphere and Atmosphere Experiment in the Amazon) pela disponibilidade dos dados.

\section{Referências}

AVISSAR R, NOBRE C. A preface to special issue on the large-scale biosphere-atmosphere experiment in Amazonia (LBA). J. Geophys. Res. 2002;107(D20):8034.

CHEN Y, XIA J, LIANG S, FENG J, FISHER JB, LI X. et al. Comparison of satellite-based evapotranspiration models over terrestrial ecosystems in china. Remote Sens. Environ. 2014; 140:279-293.

HU G, JIA L, MENENTI M, Comparison of MOD16 and LSA-SAF MSG evapotranspiration products over Europe for 2011. Remote Sens. Environ. 2015; 156:510-526.

KIM HW, HWANG K, MU Q, LEE SO, CHOI M. Validation of MODIS 16 global terrestrial evapotranspiration products in various climates and land cover types in Asia. KSCE J. Civil Eng. 2012;16(2):229-238.

LIAQAT UW, CHOI M. Accuracy comparison of remotely sensed evapotranspiration products and their associated water stress footprints under different land cover types in Korean peninsula. J. Clean. Prod. 2017; 155:93-104.

MARTENS B, MIRALLES D G, LIEVENS H, VAN DER SCHALIE R, DE JEU R A, FERNANDEZ-PRIETO D. et al. Gleam v3: Satellite-based land evaporation and root-zone soil moisture. Geoscientific Model Development. 2017;10(5):1903-1925.

MIRALLES D, HOLMES T, DE JEU R A, GASH J, MEESTERS A, DOLMAN A. Global landsurface evaporation estimated from satellite-based observations. Hydrology and Earth System Sciences. 2011;15(2): 453-469.

MU, Q.; HEINSCH, F. A.; ZHAO, M.; RUNNING, S. W. Development of a global evapotranspiration algorithm based on modis and global meteorology data. Remote Sens. Environ. 2007;111(4):519-536.

MU Q, ZHAO M, RUNNING SW. Improvements to a MODIS global terrestrial evapotranspiration algorithm. Remote Sens. Environ.2011;115(8):1781-1800.

OLIVEIRA G, BRUNSELL NA, MORAES EC, SHIMABUKURO YE, BERTANI G, SANTOS TV. et al. Evaluation of MODIS-based estimates of water-use efficiency in Amazonia. Int. J. Remote Sens. 2017;38(19):5291-5309.

ROBERTS DA, KELLER M, SOARES JV. Studies of land-cover, land-use, and biophysical properties of vegetation in the large scale biosphere atmosphere experiment in Amazônia. Remote Sens. Environ. 2003;87(4):377-388.

RUHOFF A, PAZ A, ARAGAO L, MU Q, MALHI Y, COLLISCHONN W. et al. Assessment of the MODIS global evapotranspiration algorithm using eddy covariance measurements and hydrological modelling in the Rio Grande basin. Hydrolog. Sci. J. 2013;58(8):1658-1676.

RUHOFF AL. Sensoriamento remoto aplicado à estimativa da evapotranspiração em biomas tropicais [thesis]. Universidade Federal do Rio Grande do Sul: Instituto de Pesquisas Hidráulicas/IPH; 2011. 180 p.

WILSON K, GOLDSTEIN A, FALGE E, AUBINET M, BALDOCCHI D, BERBIGIER P. et al. Energy balance closure at FLUXNET sites. Agric. For. Meteorol. 2002;113(1):223-243. 
Adriana Aparecida Moreira

Instituto de Pesquisa Hidráulicas - Universidade Federal do Rio Grande do Sul E-mail: moreiradriana00@gmail.com

Daniela Santini Adamatti

Instituto de Pesquisa Hidráulicas - Universidade Federal do Rio Grande do Sul E-mail: dani.adamatti@gmail.com

Anderson Luis Ruhoff

Instituto de Pesquisa Hidráulicas - Universidade Federal do Rio Grande do Sul E-mail: andersonruhoff@gmail.com 